Article

\title{
In Vitro Antimicrobial and Antioxidant Activities of Ethanolic Extract of Lyophilized Mycelium of Pleurotus ostreatus PQMZ91109
}

\section{Emanuel Vamanu}

Faculty of Biotechnology, University of Agronomical Sciences and Veterinary Medicine Bucharest, 59 Marasti blvd, Bucharest 011464, Romania; E-Mail: email@emanuelvamanu.ro

Received: 23 February 2012; in revised form: 19 March 2012 / Accepted: 21 March 2012 /

Published: 26 March 2012

\begin{abstract}
The antioxidant and antimicrobial potential of the ethanolic extract of Pleurotus ostreatus PQMZ91109 mycelium was determined based on inorganic and organic nitrogen sources in the culture medium. The presence of ammonium sulfate resulted in a greater accumulation of bioactive compounds compared with the organic ones. This finding was also confirmed by the low values of the ascertained $\mathrm{EC}_{50}$ and minimum inhibitory concentration (MICs). Among the organic sources, peptone followed by corn extract, led to a more important radical-scavenging activity. The extracts selectively inhibited the tested strains, mainly the two of the genus Candida, at an MIC value of $1.25 \mathrm{mg} / \mathrm{mL}$. The antioxidant potential was evaluated by the inhibition capacity of the 2,2-diphenyl-1-picrylhydrazyl (DPPH) radical, $\beta$-carotene-linoleic acid, which is the reducing power. In addition, the quantity of the compounds with antioxidant effects confirmed the data obtained, they being present in the extracts.
\end{abstract}

Keywords: mushroom; mycelium; antimicrobial; antioxidant; phenols; flavonoids

\section{Introduction}

Edible mushrooms grow spontaneously on tree trunks or on decaying woody debris, in places with high humidity. Shiitake mushrooms such as Lentinus edodes, Maitake mushrooms such as Grifola frondosa, chanterelles such as Cantharellus cibarius, white button mushrooms such as Agaricus bisporus, and oyster mushrooms have shown that they serve as repositories of B-vitamins, organic acids, $\beta$-glucans, lipids, proteins, and micronutrients such as selenium or chromium [1]. Studies 
conducted to date reveal that the antimicrobial, antioxidant, and antitumor effects are due to the presence of these substances in aqueous or alcoholic extracts [2]. A special category of bioactive compounds is represented by the polysaccharides synthesized by these fungi. The obvious therapeutic effects of polysaccharides of the $\beta$-glucan type have emerged after undergoing cultivation in liquid medium [3]. Significant antioxidant effects of extracts from the mycelia of some mushrooms like Coprinus comatus, Pleurotus ostreatus, and Leucopaxillus giganteus were also highlighted by cultivation in liquid medium [4-6]. Consequently, it was recommended that the fungi and their derivatives can be used not only as dietary supplements, but also in medicinal products. Such products are used as an alternative to prevent and even treat diseases caused by the current lifestyle of mankind.

Pleurotus mushrooms, commonly known as oyster mushrooms, are quite easily cultivated artificially, most often in liquid medium. The oyster mushroom $P$. ostreatus is appreciated as a food due to its flavor and for its medicinal and bioremediational properties [7]. The species of the Pleurotus genus are considered to be an important source of dietary fiber and contain other important nutrients. Additionally, the antioxidant effect and the capacity to inhibit free radicals derive from the amount of phenolic compounds and flavonoids they contain [8,9]. Lyophilized extracts from P. ostreatus mycelium possess antioxidant and reducing activities which are higher than those of other commercial mushrooms. The antioxidant activities were positively correlated with the total polyphenol content, supported by lyophilization, a procedure that retains the highest quantities of these compounds [10]. Generally, freeze drying retains higher levels of phenolic content in samples than air drying $[11,12]$. The observed antimicrobial activity was classified as a defense mechanism against other organisms.

In the present study, the influence of an inorganic nitrogen source (ammonium sulfate) and of three organic sources (peptone, yeast extract, and corn extract) on the production of mycelium and on its functional properties was evaluated by determining the antioxidant activity, the reduction power, the inhibition of free radicals, and the antimicrobial activity. The antimicrobial activity was highlighted against some potentially pathogenic strains of Gram-positive and Gram-negative bacteria, as well as against two strains of the genus Candida. Also, determination of the amount of phenolic compounds, of flavonoids, and of the carotenoid compounds ( $\beta$-carotene and lycopene) was carried out.

\section{Results and Discussion}

\subsection{Effect of Nitrogen Source on Mycelia Growth}

Use of an inorganic nitrogen source, ammonium sulfate, led to the best results, i.e., $22.77 \mathrm{~g}$ mycelium/L. For the three sources of organic nitrogen the order was: corn extract $>$ peptone $>$ yeast extract (Figure 1). In general, it can be appreciated that PQMZ91109 development was compact, with the density of the hyphae depending on the source of nitrogen used. It was observed that in the presence of corn extract the color of the hyphae was darker, more towards brown. In the other cases, the color was whitish - creamy. The difference between the presence of ammonium sulfate and corn extract in the culture medium was approximately 14\%. The size of the colonies was small with an average diameter of less than $0.1 \mathrm{~cm}$ when ammonium sulfate and corn extract were used. Peptone and yeast extract are also known to be efficient nitrogen sources for fungal biomass production but essentially, the quantity obtained depends on the used strain [13]. A smaller colony diameter could be 
explain by the development of high mycelia density that, probably, consumes more time. A medium containing less nutrients prompts the colony to grow rapidly in search of nutrition [14].

Figure 1. Effect of nitrogen source on mycelia growth of the Pleurotus ostreatus PQMZ91109. Values are expressed as mean $\pm \mathrm{SD}(n=3)$.

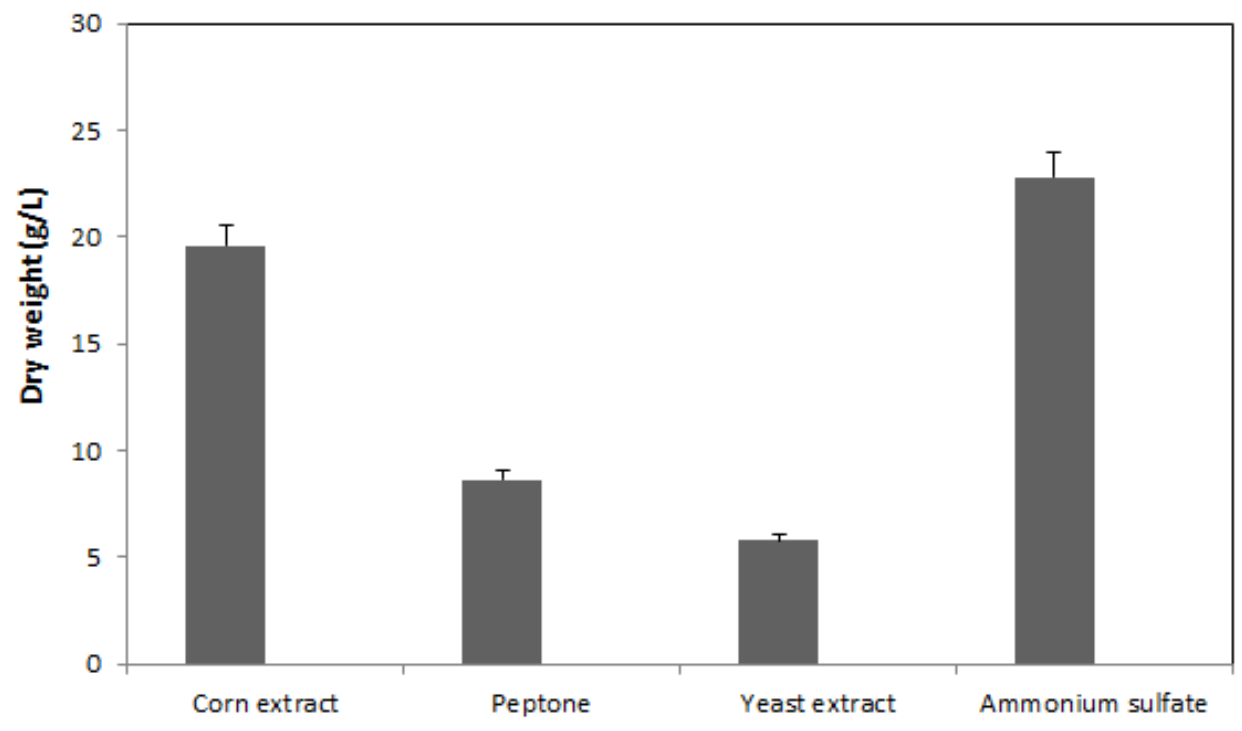

Edible mushroom mycelium possesses a high antioxidant capacity. Current research is focused on determining the conditions for advantageous cultivation in order to obtain a mycelium possessing significant biological and pharmacological activities. The study performed on PQMZ91109 mycelium growing in submerged conditions is supported by other previous studies that were conducted under similar conditions, with special reference to the nitrogen source [15], to the stirring conditions, and to the length of cultivation [16]. These studies confirm that the inorganic nitrogen was the most suitable nitrogen source. Of the organic ones, the use of peptone is confirmed, but in addition, the study highlights the possible use of corn extract as a source of nitrogen that can be used to obtain mycelium with high antioxidant activity. As regards the peptone, the studies, likewise, confirmed the presence of strains such as Coriolus versicolor [17]. Ease of use and low cost price make it an ideal source of nitrogen for cultivation in bioreactors. Use of corn extract is also confirmed by its heterogeneous composition, which in addition to various growth factors, vitamins, contains a small amount of carbohydrates, of which sucrose registers the largest proportion [18].

\subsection{Scavenging Effect on DPPH}

Stable DPPH radicals are widely used to evaluate the antioxidant activities of proton-donating substances according to their hydrogen-donating ability. DPPH radicals accept electrons or hydrogen radicals to form stable diamagnetic molecules. The antioxidant activity of substances can be expressed as the reduction capability of DPPH radicals at $517 \mathrm{~nm}[19,20]$. The scavenging activity of radicals increased with increasing percentage of free radical inhibition [21]. The degrees of discoloration caused by the loss of the initial purple showed the potential for binding of the free radicals. The scavenging effect of the ethanolic freeze-dried PQMZ91109 mycelium on DPPH radicals increases with sample concentration, depending on the source of nitrogen used for the cultivation of the 
mycelium (Figure 2). Maximum values were obtained for mycelium grown in the presence of ammonium sulfate. The obtained order, depending on the source of nitrogen was: ammonium sulfate $>$ peptone $>$ corn extract $>$ yeast extract. The scavenging activity of DPPH at a concentration of maximum $20 \mathrm{mg} / \mathrm{mL}$ ranged between 58.76 and 89.93\%. Thus, the $\mathrm{EC}_{50}$ was $6.74 \mathrm{mg} / \mathrm{mL}$ for ammonium sulfate. In addition, ascorbic acid revealed a lower scavenging ability of $45.97 \%$, at $20 \mathrm{mg} / \mathrm{mL}$.

Figure 2. Scavenging activity of extracts from the Pleurotus ostreatus PQMZ91109 mycelium against 1,1-diphenyl-2-picrylhydrazyl. Values are expressed as mean $\pm \operatorname{SD}(n=3)$.

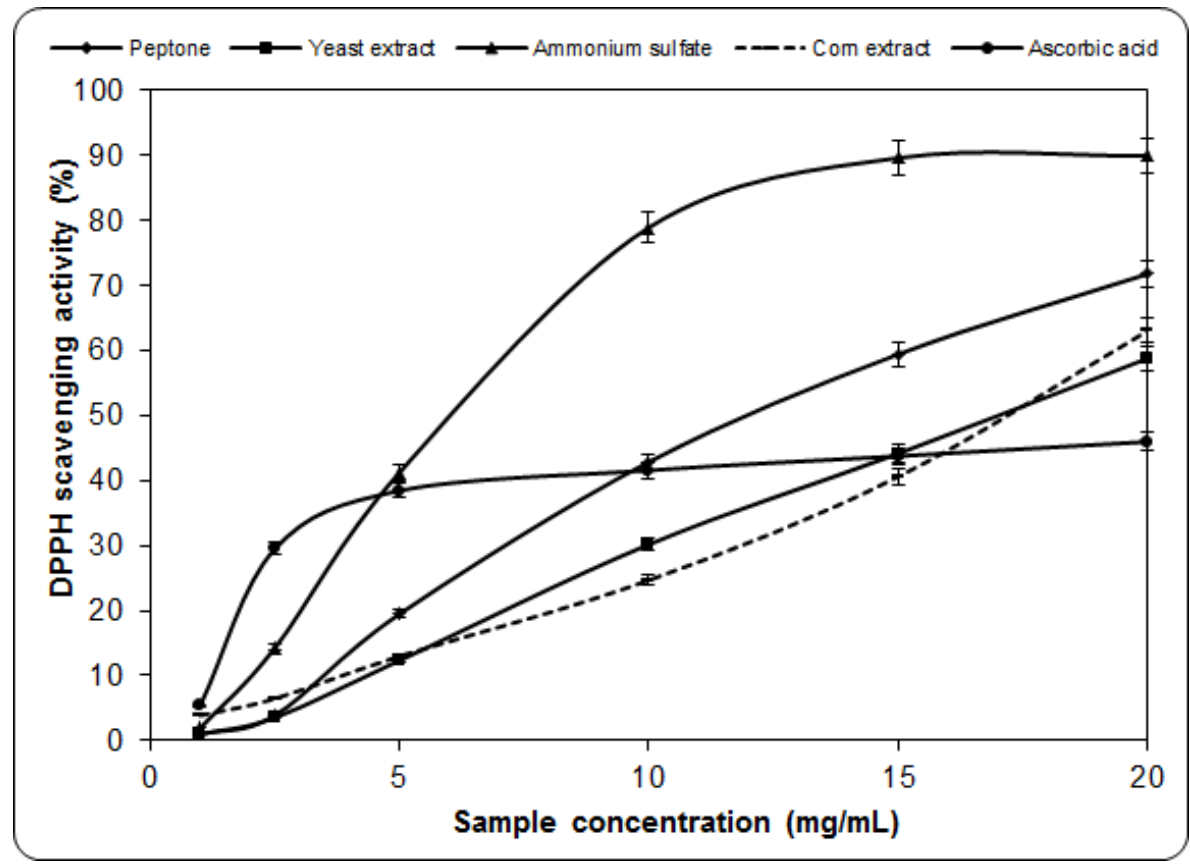

In the case of Pleurotus squarrosulus, similar percentages of DPPH scavenging activity, of 80-85\% for aqueous extracts have been reported. In turn, the methanolic extract showed similar values. For PQMZ91109, these values are dependant on the nitrogen source. On the other hand, for Agaricus blazei, a scavenging ability of $97.4 \%$ is obtained, which means a difference of $7.6 \%$ [6] when compared with the maximum value obtained for PQMZ91109, at $20 \mathrm{mg} / \mathrm{mL}$. For the ethanolic extracts of G. frondosa T1 and T2, the scavenged DPPH radicals were 99.19 and $84.36 \%$ at $20 \mathrm{mg} / \mathrm{mL}$ [22]. For Leucoagaricus pudicus, DPPH scavenging activity was $64.6 \%$, and for Amanita caesarea, it was $79.4 \%$ in both cases for the same concentration of $20 \mathrm{mg} / \mathrm{mL}$. Thus, it may be safely considered that for these species, both the alcoholic and aqueous extracts gave rise to the presence of significant amounts of antioxidant compounds. They react with DPPH radicals, the majority of them being reduced. The performance of ethanolic extracts of PQMZ91109 was higher than the standard ascorbic acid, which is in agreement with some previous studies $[23,24]$.

\subsection{Antioxidant Activity Against $\beta$-Carotene-Linoleic Acid}

Polyunsaturated fatty acids, such as linoleic acid, are easily oxidized by oxygen in air. This auto-oxidation leads to the occurrence of chain reactions with the formation of coupled double bonds, and at a later stage also obtaining secondary products such as aldehydes, ketones, and alcohols [8,25]. Using this method to characterize the antioxidant capacity it was noticed that it increases with sample 
concentration. The most effective source of nitrogen was the inorganic one, ammonium sulfate. For the organic ones the order was: corn extract $>$ peptone $>$ yeast extract (Figure 3). If the $\mathrm{EC}_{50}$ is about $9.8 \mathrm{mg} / \mathrm{mL}$ for ammonium sulfate, for the corn extract it increases up to $13.8 \mathrm{mg} / \mathrm{mL}$. In contrast, for BHT, the value is $0.2 \mathrm{mg} / \mathrm{mL}$. The inhibition ratio corresponds to the previous studies of Barros et al. [4], which showed that the antioxidant activities of L. giganteus and Agaricus arvensis were 61.4 and $46.7 \%$ for concentrations below $10 \mathrm{mg} / \mathrm{mL}$. Instead, the antioxidant activity of PQMZ91109 was $64.12 \%$ at $10 \mathrm{mg} / \mathrm{mL}$ for ammonium sulfate. The obtained values were outweighed by extracts from the fruiting bodies of $L$. edodes which had a value of more than $90 \%$ at a concentration of $8 \mathrm{mg} / \mathrm{mL}$ [26].

Figure 3. Antioxidant activity against $\beta$-carotene-linoleic acid of ethanolic extracts from Pleurotus ostreatus PQMZ91109. Values are expressed as mean $\pm \operatorname{SD}(n=3)$.

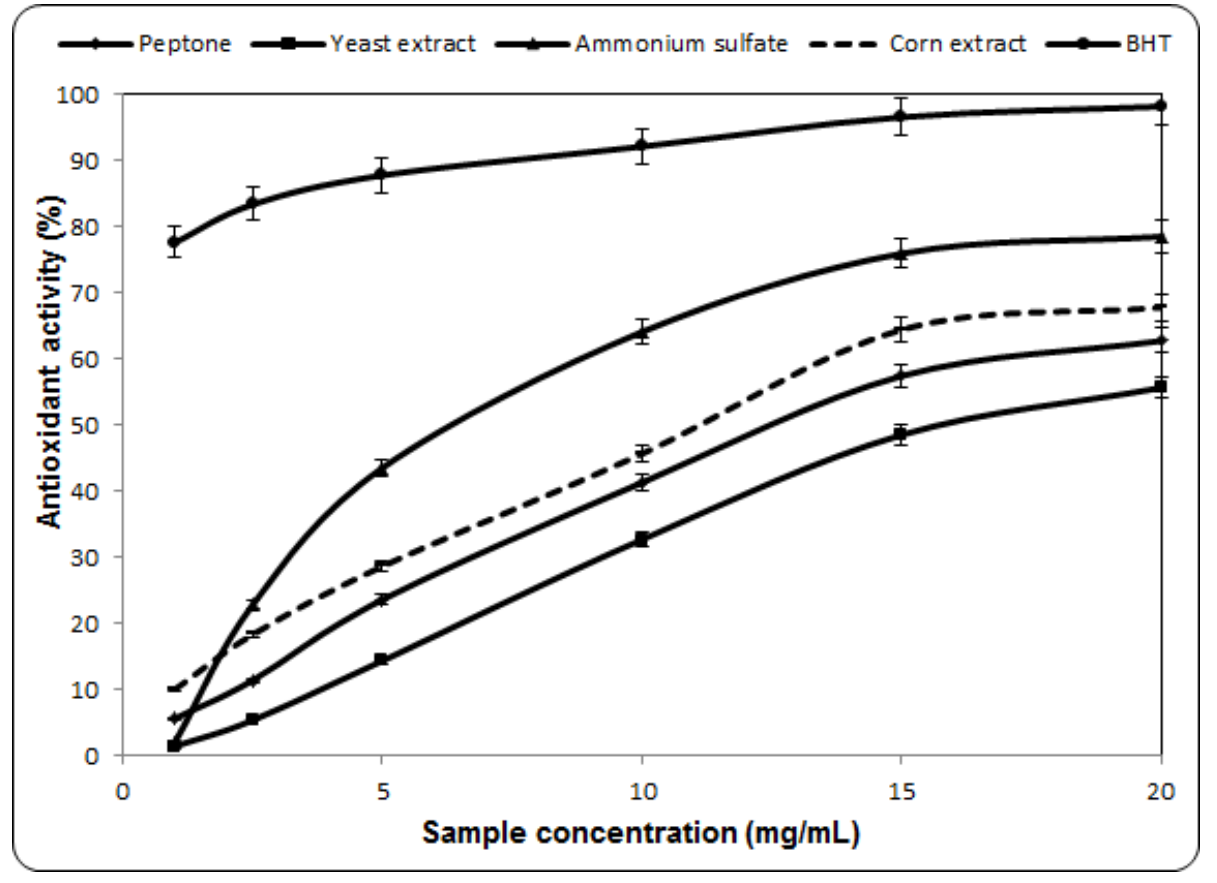

\subsection{Determination of Reducing Power}

In the reducing power assay, most antioxidant compounds convert the oxidized form of iron $\left(\mathrm{Fe}^{3+}\right)$ in ferric chloride to ferrous $\left(\mathrm{Fe}^{2+}\right)$ [27]. This is because the reducing capacity of a compound may serve as a significant indicator of its antioxidant potential, and the efficacy of certain antioxidants is known to be associated with their reducing power [5].

The results of this research showed that the reducing power of lyophilized mycelium obtained in the presence of ammonium sulfate yielded the best results (Figure 4), with a maximum value of 1.49 for a concentration of $20 \mathrm{mg} / \mathrm{mL}$. From the resulting data obtained it was found that all extracts possess reduction capacity. $\mathrm{EC}_{50}$ values for the reducing power were $5.24,7.7,6.37$, and $3.85 \mathrm{mg} / \mathrm{mL}$ for peptone, yeast extract, corn extract, and ammonium sulfate, respectively. In contrast, for ascorbic acid, the value was $0.085 \mathrm{mg} / \mathrm{mL}$. Compared with L. edodes dry extracts, they were higher by about $60 \%$ for a $1 \mathrm{mg} / \mathrm{mL}$ concentration. Compared with the extracts of Hericium erinaceum, the difference was about $48 \%$ for the same concentration [28]. Thus, the obtained results indicated the presence of some significant amounts of reductone and ascorbic acid, which could react with free radicals to stabilize 
and block radical chain reactions. Compared with previous researches using the same species [5], valuable results have been obtained, which represent additional confirmation of the fact that the nitrogen source has a major influence not only on the amount of mycelium produced in liquid medium, but also on antioxidant properties of the alcoholic extracts.

Figure 4. Reducing power of ethanolic extracts from the mycelium of Pleurotus ostreatus PQMZ91109. Values are expressed as mean $\pm \operatorname{SD}(n=3)$.

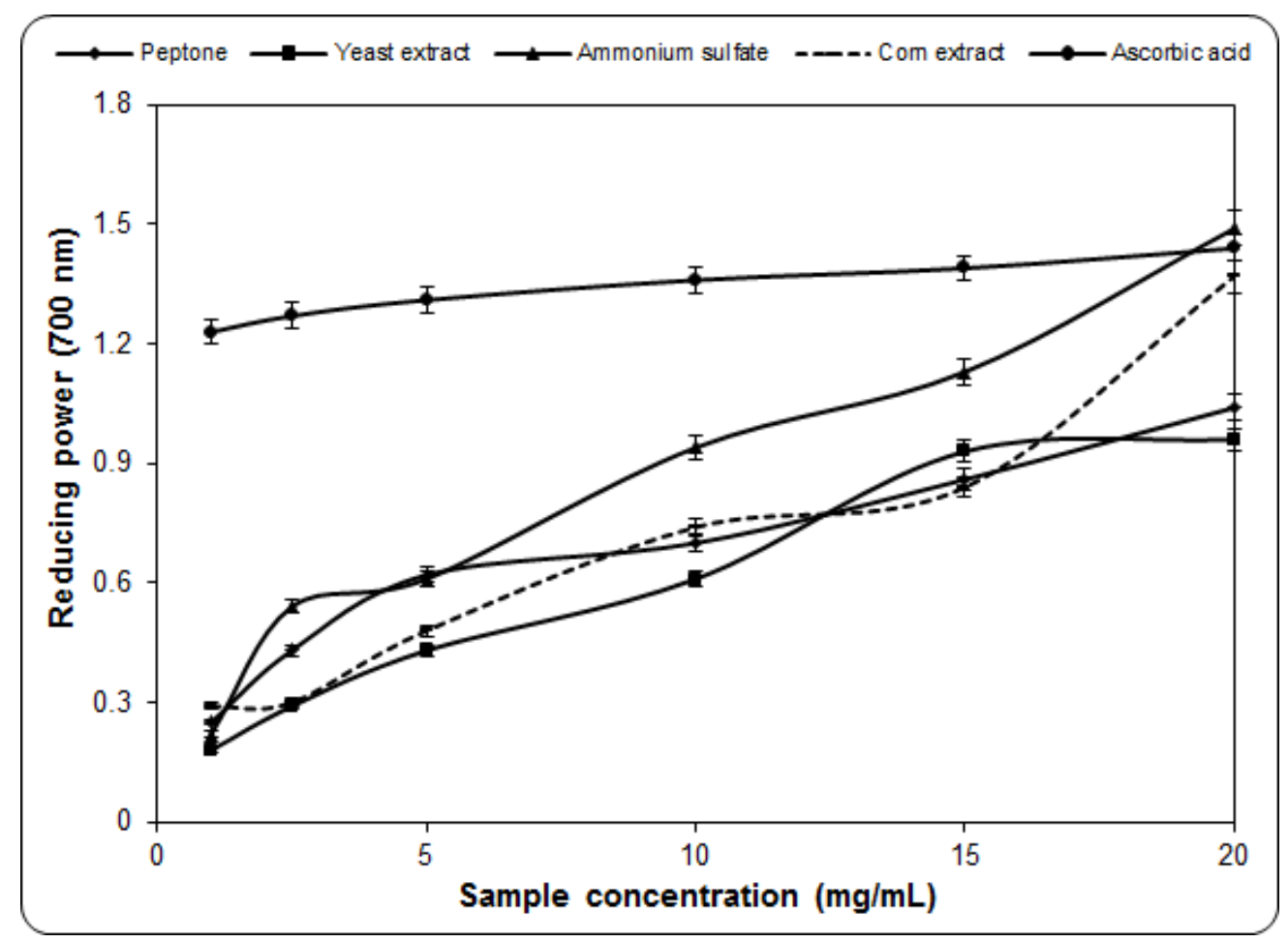

\subsection{Scavenging Effect on Hydroxyl Radicals}

The hydroxyl radical is the most reactive of the reactive oxygen species, and it induces severe damage in adjacent biomolecules. The hydroxyl radical can cause oxidative damage to DNA, lipids, and proteins [5]. Thus, removing the hydroxyl radical is vital for the protection of living systems [29]. The ethanol extracts from PQMZ91109 mycelium display a scavenging activity according to concentration and nitrogen source, as shown in Table 1. Following the studies conducted, the hydroxyl radical-scavenging effect of the mushroom extract in a concentration of $20 \mathrm{mg} / \mathrm{mL}$ was $81.5 \%$ for the mycelium obtained in the presence of inorganic nitrogen source. In general, all extracts showed good hydroxyl scavenging activity. $\mathrm{EC}_{50}$ values for peptone and corn extract were very close with a difference of about $30 \%$ of that determined for ascorbic acid at $6 \mathrm{mg} / \mathrm{mL}$ used as the standard. Instead, for ammonium sulfate, the difference when compared with the standard was approximately $9 \%$. The obtained data are consistent with those reported for the strain $P$. squarrosulus in the case of methanolic extract, but not for the aqueous ones where the $\mathrm{EC}_{50}$ value could drop by $51 \%$ [30]. For strains of the same species, the values of $\mathrm{EC}_{50}$ can reach $8 \mathrm{mg} / \mathrm{mL}$ [5] which is higher by about $30 \%$, and which demonstrates the efficiency of mycelium produced by submerged culture. 
Table 1. $E_{50}$ values for radical scavenging effect and bioactive compounds obtained from different mycelium extracts of Pleurotus ostreatus PQMZ91109. Values are mean \pm SD of 3 separate determinations, each in triplicate.

\begin{tabular}{|l|l|l|l|l|}
\hline & \multicolumn{4}{|c|}{ EC $_{\mathbf{5 0}}(\mathbf{m g} / \mathbf{m L})$} \\
\cline { 2 - 5 } & Peptone & Yeast extract & Ammonium sulfate & Corn extract \\
\hline Hydroxyl scavenging activity & $8.81 \pm 0.06$ & $14.47 \pm 0.11$ & $6.54 \pm 0.04$ & $8.54 \pm 0.02$ \\
\hline $\begin{array}{l}\text { Superoxide radical } \\
\text { scavenging activity }\end{array}$ & $2.81 \pm 0.18$ & $4.71 \pm 0.2$ & $1.27 \pm 0.86$ & $1.65 \pm 0.61$ \\
\hline Nitric oxide scavenging activity & $1.34 \pm 0.4$ & $3.78 \pm 0.59$ & $0.77 \pm 0.47$ & $1.22 \pm 0.85$ \\
\hline & \multicolumn{4}{|c|}{ Bioactive Compounds } \\
\hline Ascorbic acid (mg/100 g) & $19.34 \pm 0.1$ & $17.8 \pm 0.05$ & $20 \pm 0.07$ & $21 \pm 0.02$ \\
\hline $\begin{array}{l}\text { Total free phenolics (mg gallic } \\
\text { acid/100 g) }\end{array}$ & $80 \pm 0.12$ & $66 \pm 0.43$ & $83 \pm 0.1$ & $71 \pm 0.51$ \\
\hline Flavonoids (mg quercetin/100 g) & $450 \pm 0.79$ & $387 \pm 0.41$ & $531 \pm 0.54$ & $477 \pm 0.3$ \\
\hline Lycopene (mg/100 g) & - & $0.048 \pm 0.02$ & $0.27 \pm 0.02$ & $0.32 \pm 0.06$ \\
\hline $\boldsymbol{\beta}$-carotene (mg/100 g) & - & - & $0.32 \pm 0.12$ & $0.44 \pm 0.1$ \\
\hline $\boldsymbol{\alpha}$-tocopherol (mg/100 g) & $1.57 \pm 0.58$ & $1.8 \pm 0.22$ & $18.76 \pm 1.07$ & $28.59 \pm 0.61$ \\
\hline
\end{tabular}

\subsection{Scavenging Effect on Superoxide Radicals}

Scavenging effect on superoxide radicals is important because it plays a significant role in the generation of other reactive oxygen species such as hydrogen peroxide, hydroxyl radical, or singlet oxygen in living systems [31,32]. The superoxide anions are free radicals common in living systems, being generated by auto-oxidation processes or by enzymes [33-35]. The results obtained, presented as $\mathrm{EC}_{50}$ index (Table 1), show that ethanol extracts from PQMZ91109 mycelium manifest inhibition on the generated superoxide $(p<0.05$ ). It reaches up to $82.4 \%$ for ammonium sulfate for a sample of $20 \mathrm{mg} / \mathrm{mL}$. For the corn extract and peptone, the $\mathrm{EC}_{50}$ value increased by about 30 and $55 \%$, respectively. Again, yeast extract was the source of nitrogen that displayed the lowest scavenging effect. Comparing the obtained results with those of the standard for vitamin $\mathrm{C}$ resulted in PQMZ91109 mycelium extract exhibiting a strong scavenging activity. As standard, the $\mathrm{EC}_{50}$ was lower with approximately 34\%. The obtained data correspond to previous results obtained with recognized medicinal herbs, for example, extracts from seeds of Psoralea corylifolia. The ethanol-water extract had a capacity of superoxide radical inhibition of 83.3\% [34]. Compared with ethanolic extracts of the algae Ulva fasciata and Gracilaria salicornia, the results were similar, except those for which the sample concentration was at half strength [36].

\subsection{Scavenging Activity of Nitric Oxide}

A high concentration of nitric oxide is associated with many diseases. Oxygen can react with nitric oxide leading to the appearance of nitrite and proxy nitrite anions which are other categories of free radicals [37]. Ethanolic extracts showed a strong inhibition effect of nitric oxide, with a maximum of $96.31 \%$ for the extract of mycelium grown in the presence of inorganic nitrogen source $(p<0.05)$. For organic nitrogen sources, the obtained order, depending on the scavenging activity, was: corn 
extract $>$ peptone $>$ yeast extract. Thus, the concentration of the mycelium extract, which achieved a $50 \%$ inhibition, was approximately $0.77 \mathrm{mg} / \mathrm{mL}$ for the ammonium sulfate. Instead, according to the presented order, the increase in the sample concentration was approximately $37,42.5$, and $80 \%$, respectively. Root extracts of Hedranthera barteri presented an $\mathrm{EC}_{50}$ value of $0.47 \mathrm{mg} / \mathrm{mL}$, and the curcumin, used as standard, presented a value of $0.04 \mathrm{mg} / \mathrm{mL}$ [38]. Compared with Moroccan truffles, where the $\mathrm{EC}_{50}$ was $0.25 \mathrm{mg} / \mathrm{mL}$, in the case of the extract from PQMZ91109 lyophilized mycelium it was at least $67.5 \%$ higher for ammonium sulfate and with a minimum $79.5 \%$ for organic nitrogen sources [39].

\subsection{Effect of Nitrogen Source on Antimicrobial Activity}

The antimicrobial susceptibility testing conducted against target microorganisms is presented in Table 2. In general, the mycelium obtained in the presence of the four nitrogen sources showed a strong antimicrobial activity. Differences are also due to the type of organism tested. This low value of MIC indicates that these components possess promising potential to inhibit some of the antibiotic-resistant bacteria [40].

Table 2. Antimicrobial activity of mycelia extracts obtained by using four different nitrogen sources. Values are mean \pm SD of 3 separate determinations, each in triplicate.

\begin{tabular}{|c|c|c|c|c|c|c|c|}
\hline \multirow{2}{*}{$\begin{array}{c}\text { Nitrogen } \\
\text { source }\end{array}$} & $\begin{array}{c}\text { Escherichia } \\
\text { coli } \\
\text { CBAB 2 }\end{array}$ & $\begin{array}{c}\text { Bacillus } \\
\text { cereus } \\
\text { CMGB } \\
\mathbf{2 1 5}\end{array}$ & $\begin{array}{c}\text { Listeria } \\
\text { innocua } \\
\text { CMGB } \\
\mathbf{2 1 8}\end{array}$ & $\begin{array}{c}\text { Candida sp. } \\
\text { ICCF15 }\end{array}$ & $\begin{array}{c}\text { Candida } \\
\text { albicans } \\
\text { ATCC } \\
\mathbf{2 0 2 3 1}\end{array}$ & $\begin{array}{c}\text { Pseudomonas } \\
\text { aeruginosa } \\
\text { ATCC 15442 }\end{array}$ & $\begin{array}{c}\text { Staphylococcus } \\
\text { aureus } \\
\text { ATCC 6588 }\end{array}$ \\
\hline Corn extract & 1.25 & 12.5 & 20 & 1.25 & 1.25 & 12.5 & 12.5 \\
\hline $\begin{array}{c}\text { Ammonium } \\
\text { sulfate }\end{array}$ & - & 2.5 & 2.5 & 1.25 & 1.25 & 2.5 & 12.5 \\
\hline Yeast extract & - & 2.5 & 20 & 1.25 & 1.25 & 20 & 12.5 \\
\hline Peptone & - & 5 & 2.5 & 1.25 & 1.25 & 2.5 & 12.5 \\
\hline
\end{tabular}

The results were recorded as presence or absence of inhibition zones. The inhibitory zones indicated the absence of bacterial growth reported as positive, and the absence of the inhibitory zone reported as negative [41,42]. The two yeast strains were strongly inhibited by all extract types, resulting in an MIC value of $1.25 \mathrm{mg} / \mathrm{mL}$. Escherichia coli $\mathrm{CBAB} 2$ proved to be resistant to these extracts, except that which was obtained from mycelium grown in the presence of corn extract. Staphylococcus aureus ATCC 6588 was found to require a concentration of $12.5 \mathrm{mg} / \mathrm{mL}$ extract, regardless of the source of nitrogen used, for the occurrence of high antimicrobial activity. In contrast, Pseudomonas aeruginosa ATCC 15442 and Listeria innocua CMGB 218 were strongly inhibited by the mycelium extract grown in the presence of peptone and ammonium sulfate. In general, the inorganic nitrogen source, ammonium sulfate, resulted in obtaining a mycelium of which ethanolic extract has shown the most pronounced antimicrobial effect. The obtained results are contrary to those presented by Barros et al. [4] regarding the mycelium of L. giganteus, the extract of which requires a concentration of at least $20 \mathrm{mg} / \mathrm{mL}$ to inhibit the growth of yeasts of the genus Candida. Instead, they are in agreement with the findings regarding $E$. coli strain which also possesses a high resistance requiring a minimum of $20-25 \mathrm{mg} / \mathrm{mL}$ in the mentioned study. 


\subsection{Antioxidant Components}

Table 1 presents the quantities of the most important components of fungi extracts that had a major contribution to the antioxidant effect and antiradical activities. Ascorbic acid is reported to directly interact with radicals in plasma, thus preventing damage to red cell membranes [5]. In this case, the difference was determined by the nitrogen source used for mycelium growth.

Thus, the amount of ascorbic acid ranged from 17.8 to $21 \mathrm{mg} / 100 \mathrm{~g}$ of freeze-dried extract. The obtained amount was significant for this genus, in which a maximum of $25 \mathrm{mg} / 100 \mathrm{~g}$ was achieved [5]. Compared with species of the genus Agaricus and L. edodes, the results were similar, falling within the presented limits [43]. Compared with the studies of Barros et al. [4] regarding wild edible Portuguese mushrooms, PQMZ91109 content can be lower at 44\% [10].

Phenolic compounds derived from plants are a favorite research target because of their possible use as dietary supplements or food preservatives [5]. In the case of mushrooms, they represent the main antioxidant component. For PQMZ91109, the obtained amount was very high, being double compared to that obtained for L. giganteus strain in the presence of $\mathrm{KNO}_{3}$ as an inorganic source of nitrogen [44]. Compared with ethanol extracts from $P$. ostreatus freshly harvested whole mushrooms, in the case of the mycelium obtained in liquid medium the total amount of phenols was at least 10 times higher [5]. The minimum quantity of $66 \mathrm{mg}$ gallic acid/100 g was obtained for yeast extract. The maximum accumulated amount of $83 \mathrm{mg}$ gallic acid/100 g, was obtained for ammonium sulfate. In this case, the total quantities of phenols obtained were higher even in comparison with Ganoderma lucidum, which was $55.96 \mathrm{mg} / \mathrm{g}$ [45].

The amount of flavonoids in the mycelium extracts obtained in the presence of four different nitrogen sources, confirmed that the inorganic source, ammonium sulfate, provided the best results. For the organic source, the order was: corn extract $>$ peptone $>$ yeast extract $(p<0.05)$. The results correspond to those previously carried out by Ameer and Al-Laith [39] with Tirmania nivea. The obtained values correspond to some previous studies with $L$. edodes, where an amount of $410 \mathrm{mg} / \mathrm{g}$ was reported [46]. Compared with extracts of some medicinal herbs like Echinacea purpurea L, with $86 \mathrm{mg}$ quercetin/g, the obtained quantities were a consequence of the growing PQMZ91109 mycelium in liquid medium, and of the importance of the nitrogen source [47].

Carotenoids represent a major category of antioxidants, being known to bestow health benefits. Carotenoids are important because they have an active role in the protection process of human body cells, serving to balance and offset the destructive effects of free radicals. They are also natural colorants and stabilizers [5]. PQMZ91109 mycelium extracts had a low $\beta$-carotene and lycopene content. These two carotenoid compounds were absent in the case when using peptones. Of organic nitrogen sources, only the corn extract caused the obtaining of an amount of $44 \mathrm{mg} / 100 \mathrm{~g}$ of $\beta$-carotene. This was approximately $27 \%$ higher than that in the case of ammonium sulfate. The same situation was found for lycopene, but the difference was higher at 15.6\%. Compared to studies of Jayakumar et al. [5], the results were very low, as they reported for $P$. ostreatus $\beta$-carotene amounts of $3.1 \mathrm{mg} / 100 \mathrm{~g}$. Instead, the results were in agreement with those obtained for the strain P. squarrosulus in aqueous and/or alcoholic extracts [30].

$\alpha$-Tocopherol is a classical and important antioxidant, well known as a scavenger of free radicals. It possesses anti- and pro-oxidant properties that act against low density lipoproteins, ensuring protection 
of the blood vessels and prevention of cardiovascular diseases [48]. In this study, $\alpha$-tocopherol concentration ranged from $1.57-28.59 \mathrm{mg} / 100 \mathrm{~g}$ extract. The maximum amount was identified in the mycelium extract cultivated in the presence of corn extract. Thus, it is demonstrated once again the important role of the nitrogen source in the presence of compounds with antioxidant effect.

Owing to the increasing demand for natural bioactive compounds in the pharmaceutical and food industries, the interest in fungi has risen steadily in recent years. Since the fungus mycelium contains a significant amount of vitamins, fibers, phenolic compounds, and carotenoids, research interests focused on the determination of antioxidant capacity [18]. In mushroom extracts, antioxidant capacity is mainly determined by the amount of phenolic compounds they contain. Their quantity also influences the inhibition capacity of the free radicals, which, in the case of PQMZ91109, was correlated with the used nitrogen source and compared with the extracts of other mushrooms and medicinal herbs. In addition, it was confirmed that the lyophilization of the mycelium, and mainly of the final extract, determined the preservation of the maximum amount of phenols and flavonoids, which was in agreement with studies performed on some medicinal plant extracts [15,49].

The correlation between antioxidant compounds and freeze-dried PQMZ91109 mycelia was also evaluated. The $R^{2}$ coefficient had good values between the content of total phenolics, flavonoids and ascorbic acid with antioxidant properties, depending on the nitrogen sources: for DPPH scavenging activity $\left(R^{2}=0.783-0.9606\right)$, nitric oxide scavenging activities $\left(R^{2}=0.845-0.991\right)$, superoxide radical scavenging activity $\left(R^{2}=0.788-0.9279\right)$, hydroxyl scavenging activity $\left(R^{2}=0.790-0.8979\right)$, reducing power $\left(R^{2}=0.888-0.9369\right)$ and antioxidant activity $\left(R^{2}=0.768-0.948\right)$. These data are in accordance with other researches which demonstrated the major contribution of polyphenols to the antioxidant activity of freeze-dried extracts [50]. However, the amount of carotenoids could not exhibit a positive correlation with antioxidant properties. Moreover, antioxidant activities of extracts are correlated to $\alpha$-tocopherol contents. The correlation coefficient varied from low to significant $\left(R^{2}=0.307-0.738\right)$.

The highest content of bioactive compounds of the mycelium extracts could explain the measured MIC values. A direct correlation between phenol content and antimicrobial properties was observed for all four nitrogen sources. Ammonium sulfate showed better results than organic nitrogen sources, expressed by lower values of MICs, which was in agreement with the higher content of bioactive compounds found. Because of its lower content in bioactive compounds, the mycelium cultivated in the presence of yeast extract was the less effective (higher MICs), not showing activity against Escherichia coli CBAB 2. The same behavior was observed for the other nitrogen sources, except corn extract. The correlations between antimicrobial activity and total phenolic content, expressed by $R^{2}$ values, were between 0.34 and 0.897 [51,52].

\section{Experimental}

\subsection{Chemicals}

All chemicals and reagents were purchased from Sigma Aldrich GmbH (Sternheim, Germany). All other unlabelled chemicals and reagents were of analytical grade. 


\subsection{Culture and Storage Condition}

The mushroom P. ostreatus PQMZ91109 was isolated from the stem of a poplar (Băneasa forest, Romania) and was authenticated by D. Pelinescu (Faculty of Biology, University of Bucharest, Bucharest, Romania). The mycelia were maintained on potato dextrose agar (PDA) at $4{ }^{\circ} \mathrm{C}$. The microorganisms were subcultured at regular intervals (45 days) to maintain viability.

\subsection{Media Preparation and Fermentation Condition}

The fungi were initially grown on PDA medium for 10 days at $25{ }^{\circ} \mathrm{C}$. The inoculum was prepared by growing mycelium on a LabTech rotary shaker at $150 \mathrm{rpm}$ for 5 days and at $25{ }^{\circ} \mathrm{C}$ in $500 \mathrm{~mL}$ Erlenmeyer flasks containing $250 \mathrm{~mL}$ of the following synthetic medium (per liter): $6.0 \mathrm{~g}$ glucose, $100.0 \mathrm{~g}$ malt extract, $20.0 \mathrm{~g}$ yeast extract, $1.0 \mathrm{~g} \mathrm{KH}_{2} \mathrm{PO}_{4}$, and $0.5 \mathrm{~g} \mathrm{MgSO}_{4} \times 7 \mathrm{H}_{2} \mathrm{O}$. The medium was adjusted to $\mathrm{pH} 5.5$ with $0.2 \mathrm{M} \mathrm{NaOH}$ [53].

Submerged fermentation was carried out in 1,000 mL Erlenmeyer flasks, containing $700 \mathrm{~mL}$ of liquid medium $\left(\mathrm{KH}_{2} \mathrm{PO}_{4} 0.2 \%, \mathrm{CaSO}_{4} 0.5 \%, \mathrm{MgSO}_{4} 0.05 \%\right.$, and $\mathrm{Na}_{2} \mathrm{HPO}_{4} 0.01 \%$ in $5 \%$ extract solution of corn flour) and was performed with 4 different nitrogen sources: corn extract (dry substance 40\%), peptone, yeast extract, and ammonium sulfate. The nitrogen sources were added as $10 \mathrm{~g} / \mathrm{L}$. The inoculated flasks were maintained on a LabTech rotary shaker at $150 \mathrm{rpm}$ and $25^{\circ} \mathrm{C}$. After 7 days of growth, the mycelium was recovered from the liquid medium by centrifugation at 3,500× $\mathrm{g}$ for $10 \mathrm{~min}$. Next, the obtained mycelia were washed 3 times with distilled water and freeze-dried in an Alpha 1-2 LD freeze-dryer in the absence of a cryoprotective agent $[4,54]$.

\subsection{Preparation of Mushroom Extract}

The extract was obtained by ethanol extraction of freeze-dried mushroom mycelia. Ethanol extraction of the mycelia was accomplished by stirring at $150 \mathrm{rpm}$ for $24 \mathrm{~h}$ at $20{ }^{\circ} \mathrm{C}$, with a ratio of $1 \mathrm{~g}$ of freeze-dried biomass per $10 \mathrm{~mL}$ solvent. The broth was centrifuged at 3,500× $\mathrm{g}$ for $10 \mathrm{~min}$ and the supernatant was filtered using Whatman No. 1 filter paper. The ethanol extract was freeze-dried. The freeze-dried extract was then re-dissolved in $80 \%$ ethanol $(\mathrm{v} / \mathrm{v})$ to yield solutions containing $1.0,2.5$, $5.0,10.0,15.0$, and $20.0 \mathrm{mg}$ of extract per $\mathrm{mL}$.

\subsection{Antimicrobial Activity}

In vitro antimicrobial susceptibility tests were performed using a panel of microorganisms from the collection of the Faculty of Biotechnology, Bucharest, Romania: Gram positive bacteria (Listeria innocua CMGB 218, Bacillus cereus CMGB 215, Staphylococcus aureus ATCC 6588), Gram negative bacteria (Escherichia coli CBAB 2, Pseudomonas aeruginosa ATCC 15442), and yeast (Candida albicans ATCC 20231, Candida sp. ICCF15). The yeast and bacteria were maintained in $20 \%$ glycerol and maintained at $-80{ }^{\circ} \mathrm{C}$. 


\section{Determination of Minimum Inhibitory Concentration (MIC)}

The standard agar dilution protocol with doubling dilution was used. The extract was incorporated into nutrient agar at concentrations ranging from $0.39 \mathrm{mg} / \mathrm{mL}$ to $25 \mathrm{mg} / \mathrm{mL}$. A control without the extract was also prepared. Ten $\mu \mathrm{L}$ of each test organisms, previously diluted to $10^{6} \mathrm{CFU} / \mathrm{mL}$, were used to inoculate the plates. These were incubated at $37{ }^{\circ} \mathrm{C}$ for $24 \mathrm{~h}$ in the first instance, and for another $24 \mathrm{~h}$ before the growth was observed and recorded. The minimum inhibitory concentrations (MICs) of the extract for each test microorganism were considered the agar plate with the lowest concentrations without growth [55].

\subsection{Determination of Antioxidant Activities}

3.6.1. 1,1-Diphenyl-2-picrylhydrazyl Radical Scavenging Activity of Freeze-Dried Mushroom Extracts

The reaction mixture contained $50 \mu \mathrm{L}$ of test samples (or $80 \% \mathrm{EtOH}$ as a blank) and $5 \mathrm{~mL}$ of a $0.04 \%(w / v)$ solution of DPPH in ethanol. Discoloration was measured at $517 \mathrm{~nm}$ after incubation for $30 \mathrm{~min}$. DPPH radical concentration was calculated using the following equation: DPPH scavenging effect $(\%)=\left(A_{0}-A_{P}\right) / A_{0} \times 100$, where $A_{0}$ was the absorbance of the control and $A_{P}$ was the absorbance in the sample. The actual decrease in absorption induced by the test compounds was compared with the positive controls. Ascorbic acid was used for comparison and as a positive control. The extract concentration providing $50 \%$ of free radical scavenging activity $\left(\mathrm{EC}_{50}\right)$ was calculated from the graph of the radical scavenging activity (RSA) percentage against extract concentration [56,57].

\subsubsection{Antioxidant Activity by $\beta$-Carotene-Linoleic Acid}

The antioxidant activity was determined with slight modifications of the procedure previously described by Sokmen et al. [58] Briefly, $\beta$-carotene $(0.5 \mathrm{mg})$ was dissolved in chloroform $(1 \mathrm{~mL})$ and linoleic acid $(25 \mu \mathrm{L})$ and Tween $40(200 \mathrm{mg})$ were added. Chloroform was removed using a rotary vacuum evaporator and distilled water saturated with oxygen $(100 \mathrm{~mL})$ was added with vigorous shaking. A volume of $2.5 \mathrm{~mL}$ of this reaction mixture was dispensed into test tubes and $350 \mu \mathrm{L}$ of various concentrations of the extracts were added. The absorbance was immediately measured at $490 \mathrm{~nm}$. The reaction mixture was incubated at $50{ }^{\circ} \mathrm{C}$ for $2 \mathrm{~h}$ and the absorbance was measured again. The same procedure was repeated with a synthetic antioxidant ( $\alpha$-tocopherol [TOC] and butylated hydroxytoluene [BHT]) and a control. Inhibition ratio of linoleic acid oxidation was calculated for the test samples and for the synthetic antioxidants [59].

\subsubsection{Reducing Power of Freeze-Dried Extracts}

Reducing power was determined according to the method described by Gulcin et al. [60] Each extract (in $2.5 \mathrm{~mL}$ of ethanol) was mixed with $200 \mathrm{mM}$ sodium phosphate buffer $(2.5 \mathrm{~mL}, \mathrm{pH} 6.6)$ and $1 \%$ potassium ferricyanide $(2.5 \mathrm{~mL})$, and the mixture was incubated at $50{ }^{\circ} \mathrm{C}$ for $20 \mathrm{~min}$. Next, $10 \%$ trichloroacetic acid $(2.5 \mathrm{~mL})$ was added, and the mixture was centrifuged at 3,000 $\mathrm{g}$ for $10 \mathrm{~min}$. The upper layer $(2.5 \mathrm{~mL})$ was mixed with deionized water $(2.5 \mathrm{~mL})$ and $0.1 \%$ ferric chloride $(0.5 \mathrm{~mL})$. Finally, the absorbance was measured at $700 \mathrm{~nm}$ and compared to a blank. The extract concentration 
providing 0.5 of absorbance $\left(\mathrm{EC}_{50}\right)$ was calculated from the graph of absorbance at $700 \mathrm{~nm}$ plotted against the extract concentration. Ascorbic acid was used as positive control $[9,57]$.

\subsubsection{Superoxide Radical Scavenging Activity of Freeze-Dried Extracts}

The scavenging activity on superoxide radicals was evaluated according to the methods described by Chou et al. [61]. The reaction mixture contained the same volume of $120 \mu \mathrm{M}$ PMS (phenazine methosulfate), $936 \mu \mathrm{M} \mathrm{NADH}$, freeze-dried extract, and $300 \mu \mathrm{M} \mathrm{NBT}$, in a total volume of $1 \mathrm{~mL}$ of $100 \mathrm{mM}$ phosphate buffer ( $\mathrm{pH}$ 7.4). After $5 \mathrm{~min}$ of incubation at ambient temperature, absorbance of the resulting solution was measured at $560 \mathrm{~nm}$. The superoxide radical activity was calculated as: scavenging effect $(\%)=(1-$ absorbance of sample/absorbance of control $) \times 100$. Ascorbic acid was used for comparison. $\mathrm{EC}_{50}$ value (milligram extract $/ \mathrm{mL}$ ) is the effective concentration at which hydroxyl radicals were scavenged by $50 \%$ [62].

\subsubsection{Hydroxyl Radical Scavenging of Freeze-Dried Extracts}

Hydroxyl radical scavenging was assayed as described by Varshneya et al. [63] with a slight modification. The assay is based on the quantification of the degradation product of 2-deoxyribose by condensation with thiobarbituric acid (TBA). Hydroxyl radicals were generated using the $\mathrm{Fe}^{3+}$-ascorbate-EDTA- $\mathrm{H}_{2} \mathrm{O}_{2}$ system (the Fenton reaction). The reaction mixture contained, in a final volume of $1 \mathrm{~mL}$ : 2-deoxy-2-ribose (2.8 mM), $\mathrm{KH}_{2} \mathrm{PO}_{4}-\mathrm{KOH}$ buffer $\left(20 \mathrm{mM}\right.$, pH 7.4), $\mathrm{FeCl}_{3}(100 \mu \mathrm{M})$, EDTA $(100 \mu \mathrm{M}), \mathrm{H}_{2} \mathrm{O}_{2}(1.0 \mathrm{mM})$, ascorbic acid $(100 \mu \mathrm{M})$, and various concentrations $(2-20 \mathrm{mg} / \mathrm{mL})$ of the freeze-dried extracts or reference compound. After incubation for $1 \mathrm{~h}$ at $37{ }^{\circ} \mathrm{C}$, the reaction mixture $(0.5 \mathrm{~mL})$ was added to $2.8 \%$ TCA $(1 \mathrm{~mL})$, then $1 \%$ aqueous TBA $(1 \mathrm{~mL})$ was added and the mixture was incubated at $90{ }^{\circ} \mathrm{C}$ for 15 min to develop the color. After cooling, the absorbance was measured at $532 \mathrm{~nm}$ against an appropriate blank solution. All tests were performed three times. Percentage inhibition was evaluated by comparing the test and blank solutions. $\mathrm{EC}_{50}$ value (milligram extract $/ \mathrm{mL}$ ) is the effective concentration at which hydroxyl radicals were scavenged by $50 \%$ [64].

\subsubsection{Nitric Oxide Scavenging of Freeze-Dried Extracts}

Nitric oxide scavenging activity was measured spectrophotometrically. Sodium nitroprusside $(5 \mathrm{mmol} / \mathrm{L})$ in phosphate buffered saline, $\mathrm{pH} 7.4$, was mixed with different concentrations of the extract prepared in ethanol and incubated at $25{ }^{\circ} \mathrm{C}$ for $30 \mathrm{~min}$. A control without the test compound, but with an equivalent amount of ethanol, was also used. After $30 \mathrm{~min}, 1.5 \mathrm{~mL}$ of the incubated solution was removed and diluted with $1.5 \mathrm{~mL}$ of Griess reagent (1\% sulphanilamide, $2 \%$ phosphoric acid and $0.1 \% \mathrm{~N}$-1-naphthylethylenediamine dihydrochloride). Absorbance formed during diazotization of the nitrite with sulphanilamide and subsequent coupling with $N$-1-naphthylethylenediamine dihydrochloride was measured at $546 \mathrm{~nm}$ and the percentage scavenging activity was measured with reference to the standard (curcumin). The $\mathrm{EC}_{50}$ value (milligram extract $/ \mathrm{mL}$ ) is the effective concentration at which hydroxyl radicals were scavenged by $50 \%$ [65]. 


\subsection{Determination of Antioxidant Component}

\subsubsection{Determination of Total Phenolic Content}

The content of total phenols was determined by spectrophotometry, using gallic acid as standard, according to the method described by the International Organization for Standardization (ISO) 14502-1. Briefly, an aliquot of the diluted sample extract $(1.0 \mathrm{~mL})$ was transferred in duplicate to separate tubes containing a 1/10 dilution of Folin-Ciocalteu's reagent in water $(5.0 \mathrm{~mL})$. Then, a sodium carbonate solution $(4.0 \mathrm{~mL}, 7.5 \% \mathrm{w} / \mathrm{v})$ were added. The tubes were then allowed to stand at room temperature for $60 \mathrm{~min}$ before absorbance at $765 \mathrm{~nm}$ was measured against water. The content of total phenols was expressed as gallic acid equivalents in $\mathrm{g} / 100 \mathrm{~g}$ extract. The concentration of polyphenols in samples was derived from a standard curve of gallic acid ranging from 10 to $50 \mu \mathrm{g} / \mathrm{mL}$ (Pearson's correlation coefficient: $\left.r^{2}=0.9996\right)$ [66].

\subsubsection{Determination of Total Flavonoids}

Sample $(0.25 \mathrm{~mL}$ of different concentrations of the extracts) was added to a tube containing distilled water $(1 \mathrm{~mL})$. Next, $5 \% \mathrm{NaNO}_{2}(0.075 \mathrm{~mL}), 10 \% \mathrm{AlCl}_{3}(0.075 \mathrm{~mL})$ and $1 \mathrm{M} \mathrm{NaOH}(0.5 \mathrm{~mL})$ were added sequentially at 0,5 and $6 \mathrm{~min}$. Finally, the volume of the reacting solution was adjusted to $2.5 \mathrm{~mL}$ with double-distilled water. The absorbance of the solution at a wavelength of $410 \mathrm{~nm}$ was detected using the Helios $\lambda$ spectrophotometers. Quercetin is a ubiquitous flavonoid, present in many natural extracts, used as standard to quantify the total flavonoid content. Results were expressed in microgram quercetin equivalents $/ 100 \mathrm{~g}$ extract $[67,68]$.

\subsubsection{Determination of $\beta$-Carotene and Lycopene}

For $\beta$-carotene and lycopene determination, the dried ethanolic extract (100 $\mathrm{mg}$ ) was vigorously shaken with an acetone-hexane mixture $(4: 6,10 \mathrm{~mL})$ for $1 \mathrm{~min}$ and filtered through Whatman No. 1 filter paper. The absorbance of the filtrate was measured at 453, 505, and $663 \mathrm{~nm}$. $\beta$-Carotene and lycopene content were calculated according to the following equations:

$$
\begin{gathered}
\text { lycopene }(\mathrm{mg} / 100 \mathrm{~mL})=-0.0458 \times \mathrm{A}_{663}+0.372 \times \mathrm{A}_{505}-0.0806 \times \mathrm{A}_{453} \\
\beta \text {-carotene }(\mathrm{mg} / 100 \mathrm{~mL})=0.216 \times \mathrm{A}_{663}-0.304 \times \mathrm{A}_{505}+0.452 \times \mathrm{A}_{453}
\end{gathered}
$$

The results are expressed as mg of carotenoid/g of extract [15].

\subsubsection{Determination of $\alpha$-Tocopherol}

The content was determined spectrophotometrically according to the method of Kivcak and Akay. The $\alpha$-tocopherol content in the extracts was calculated from the regression equation of the standard curve [69].

\subsection{Statistical Analysis}

All parameters for antimicrobial and antioxidant activity were assessed in triplicate, and the results were expressed as mean \pm SD values of 3 observations. The mean values and standard deviation were calculated with the EXCEL program from Microsoft Office 2010 package. 


\section{Conclusions}

The extract of an oyster mushroom mycelium (Pleurotus ostreatus) can be an effective natural antioxidant product for food and pharmaceutical industrial applications. The mushroom mycelium contains many different bioactive compounds with diverse biological activity depending on how it is obtained. Pleurotus mycelium extracts have a strong inhibiting and other beneficial or therapeutic health effects. The ethanol extract of the PQMZ91109 lyophilized mycelium and various antioxidants used proved to exhibit antioxidant activity, reducing power, and free radical inhibition properties, being dependent on the sample concentration. The same feature was also observed for antimicrobial activity, the extracts being effective against some strains of pathogenic potential for humans. The study showed that the PQMZ91109 mycelium represents a major source of phenols, flavonoids and $\beta$-carotene, acting in addition for the prevention of some diseases of modern society. The obtained results showed that the properties of nutraceuticals depend on the nitrogen source and, therefore, the PQMZ91109 mycelium extracts can be used as a rich source of antioxidants in pharmaceutical-type products.

\section{Acknowledgments}

This research was financed through the project PNCDI II CNCSIS-Human Resources, Theme 9/2010.

\section{References and Notes}

1. Iwalokun, B.A.; Usen, U.A.; Otunba, A.A.; Olukoya, D.K. Comparative phytochemical evaluation, antimicrobial and antioxidant properties of Pleurotus ostreatus. Afr. J. Biotechnol. 2007, 6, 1732-1739.

2. Lindequist, U.; Niedermeyer, T.H.J.; Julich, W. The pharmacological potentials of mushrooms. Evid. Based Complement. Alternat. Med. 2005, 2, 285-299.

3. Smith, J.E.; Rowan, N.J.; Sullivan, R. Medicinal mushrooms: A rapidly developing area of biotechnology for cancer therapy and other bioactivities. Biotechnol. Lett. 2002, 24, 1839-1845.

4. Barros, L.; Baptista, P.; Estevinho, L.M.; Ferreira, I.C.F.R. Bioactive properties of the medicinal mushroom Leucopaxillus giganteus mycelium obtained in the presence of different nitrogen sources. Food Chem. 2007, 105, 179-186.

5. Jayakumar, T.; Thomas, P.A.; Geraldine, P. In-vitro antioxidant activities of an ethanolic extract of the oyster mushroom, Pleurotus ostreatus. Innov. Food Sci. Emerg. Technol. 2009, 10, 228-234.

6. Tsai, S.Y.; Tsai, H.L.; Mau, J.L. Antioxidant properties of Coprinus comatus. J. Food Biochem. 2009, 33, 368-389.

7. Keyhani, J.; Keyhani, E.; Attar, F.; Hadizadeh, M. Anti-oxidative stress enzymes in Pleurotus ostreatus. In Proceedings of the II International Conference on Environmental, Industrial and Applied Microbiology (BioMicroWorld2007), University of Seville, Seville, Spain, 28 November-1 December 2007; World Scientific Publishing Co.: London, UK, 2009.

8. Alam, N.; Yoon, K.N.; Cha, Y.J.; Kim, J.H.; Lee, K.R.; Lee, T.S. Appraisal of the antioxidant, phenolic compounds concentration, xanthine oxidase and tyrosinase inhibitory activities of Pleurotus salmoneostramineus. Afr. J. Agric. Res. 2011, 6, 1555-1563. 
9. Alam, N.; Yoon, K.N.; Lee, T.S. Evaluation of the antioxidant and antityrosinase activities of three extracts from Pleurotus nebrodensis fruiting bodies. Afr. J. Biotechnol. 2011, 10, 2978-2986.

10. Ferreira, I.C.F.R.; Baptista, P.; Vilas-Boas, M.; Barros, L. Free-radical scavenging capacity and reducing power of wild edible mushrooms from northeast Portugal: Individual cap and stipe activity. Food Chem. 2007, 100, 1511-1516.

11. Abascal, K.; Ganora, L.; Yarnell, E. The effect of freeze-drying and its implications for botanical medicine: A review. Phytother. Res. 2005, 19, 655-660.

12. Dai, J.; Mumper, R.J. Plant phenolics: Extraction, analysis and their antioxidant and anticancer properties. Molecules 2010, 15, 7313-7352.

13. Petre, M.; Teodorescu, A.; Țuluca, E.; Bejan, C.; Andronescu, A. Biotechnology of mushroom pellets producing by controlled submerged fermentation. Rom. Biotechnol. Lett. 2010, 15, 50-55.

14. Sung, G.H.; Shrestha, B.; Han, S.K.; Kim, S.Y.; Sung, J.M. Growth and cultural characteristics of Cordyceps cardinalis collected from Korea. Mycobiology 2010, 38, 274-281.

15. Barros, L.; Cruz, T.; Baptista, P.; Estevinho, L.M.; Ferreira, I.C.F.R. Wild and commercial mushrooms as source of nutrients and nutraceuticals. Food Chem. Toxicol. 2008, 46, 2742-2747.

16. Arora, D.S.; Chandra, P. Antioxidant activity of Aspergillus fumigates. ISRN Pharmacol. 2011, 1, $1-11$.

17. Ruhul Amin, S.M.; Sarker, N.C.; Hossain, K. Optimization of in vitro culture conditions for mycelial growth of Turkey tail mushroom (Coriolus versicolor). Bangladesh J. Mushroom 2008, 2, 63-71.

18. Jayakumar, T.; Thomas, P.A.; Sheu, J.R.; Geraldine, P. In-vitro and in-vivo antioxidant effects of the oyster mushroom Pleurotus ostreatus. Food Res. Int. 2011, 44, 851-861.

19. Elmastasa, M.; Isildaka, O.; Turkekulb, I.; Temura, N. Determination of antioxidant activity and antioxidant compounds in wild edible mushrooms. J. Food Compost. Anal. 2007, 20, 337-345.

20. Yeh, J.Y.; Hsieh, L.H.; Wu, K.T.; Tsai, C.F. Antioxidant properties and antioxidant compounds of various extracts from the edible basidiomycete Grifola frondosa (Maitake). Molecules 2011, 16, 3197-3211.

21. Acharya, S.; Sahu, A.R.; Mohanta, S.R. Free radical scavenging activity of thalamus of Nymphacea stellata willd. Int. J. Pharm. Pharm. Sci. 2010, 2, 61-63.

22. Sarikurkcu, C.; Tepe, B.; Semiz, D.K.; Solak, M.H. Evaluation of metal concentration and antioxidant activity of three edible mushrooms from Mugla, Turkey. Food Chem. Toxicol. 2010, $48,1230-1233$.

23. Duan, X.J.; Zhang, W.W.; Li, X.M.; Wang, B.G. Evaluation of antioxidant property of extract and fractions obtained from a red alga, Polysiphonia urceolata. Food Chem. 2006, 95, 37-43.

24. Srinivasan, K.; Jagadish, L.K.; Shenbhagaraman, R.; Muthumary, J. Antioxidant activity of endophytic fungus Phyllosticta sp. isolated from Guazuma tomentosa. J. Phytol. 2010, 2, 37-41.

25. Alam, N.; Yoon, K.N.; Lee, K.R.; Shin, P.G.; Cheong, J.C.; Yoo, Y.B.; Shim, M.J.; Lee, M.W.; Lee, U.Y.; Lee, T.S. Antioxidant activities and tyrosinase inhibitory effects of different extracts from Pleurotus ostreatus fruiting bodies. Mycobiology 2010, 38, 295-301.

26. Yoon, K.N.; Alam, N.; Lee, J.S.; Lee, K.R.; Lee, T.S. Detection of phenolic compounds concentration and evaluation of antioxidant and antityrosinase activities of various extracts from the fruiting bodies of Lentinus edodes. World Appl. Sci. J. 2011, 12, 1851-1859. 
27. Moein, M.R.; Moein, S.; Ahmadizadeh, S. Radical scavenging and reducing power of Salvia mirzayanii subfractions. Molecules 2008, 13, 2804-2813.

28. Vidović, S.S.; Mujić, I.O.; Zeković, Z.P.; Lepojević, Ž.D.; Tumbas, V.T.; Muji, A.I. Antioxidant properties of selected Boletus mushrooms. Food Biophys. 2010, 5, 49-58.

29. Luo, A.; Fan, Y.; Luo, A. In vitro free radicals scavenging activities of polysaccharide from Polygonum Multiflorum Thunb. J. Med. Plants Res. 2011, 5, 966-972.

30. Pal, J.; Ganguly, S.; Tahsin, K.S.; Acharya, K. In vitro free radical scavenging activity of wild edible mushroom Pleurotus squarrosulus (Mont.) Singer. Indian J. Exp. Biol. 2010, 47, 1210-1218.

31. Stief, T.W. The physiology and pharmacology of singlet oxygen. Med. Hypotheses 2003, 60, $567-572$.

32. Jiao, Z.; Liu, J.; Wang, S. Antioxidant activities of total pigment extract from blackberries. Food Technol. Biotechnol. 2005, 43, 97-102.

33. Lee, J.C.; Kim, H.R.; Kim, J.; Jang, Y.S. Antioxidant activity of ethanol extract of the stem of Opuntia ficus-indica var. saboten. J. Agric. Food Chem. 2002, 50, 6490-6496.

34. Kiran, B.; Raveesha, K.A. In vitro evaluation of antioxidant potentiality of seeds of Psoralea corylifolia L. World Appl. Sci. J. 2010, 8, 985-990.

35. Valko, M.; Leibfritz, D.; Moncol, J.; Cronin, M.T.D.; Mazur, M.; Telser, J. Free radicals and antioxidants in normal physiological functions and human disease. Int. J. Biochem. Cell Biol. 2007, 39, 44-84.

36. Vijayavel, K.; Martinez, J.A. In vitro antioxidant and antimicrobial activities of two hawaiian marine limu: Ulva fasciata (Chlorophyta) and Gracilaria salicornia (Rhodophyta). J. Med. Food 2010, 13, 1494-1499.

37. Baskar, R.; Lavanya, R.; Mayilvizhi, S.; Rajasekaran, P. Free radical scavenging activity of antitumor polysaccharide fractions isolated from Ganoderma lucidum (Fr.) P. Karst. Nat. Prod. Rad. 2008, 7, 320-325.

38. Onasanwo, S.A.; Singh, N.; Olaleye, S.B.; Mishra, V.; Palit, G. Anti-ulcer and antioxidant activities of Hedranthera barteri (Hook F.) with possible involvement of $\mathrm{H}^{+}, \mathrm{K}^{+}$ATPase inhibitory activity. Indian J. Med. Res. 2010, 132, 442-449.

39. Ameer, A.; AL-Laith, A. Antioxidant components and antioxidant/antiradical activities of desert truffle (Tirmania nivea) from various Middle Eastern origins. J. Food Compost. Anal. 2010, 23, $15-22$.

40. Ameri, A.; Vaidya, J.G.; Deokule, S.S. In vitro evaluation of anti-staphylococcal activity of Ganoderma lucidum, Ganoderma praelongum and Ganoderma resinaceum from Pune, India. Afr. J. Microb. Res. 2011, 5, 328-333.

41. Panthi, M.P.; Chaudhury, R.P. Antibacterial activity of some selected folklore medicinal plants from West Nepal. Sci. World 2006, 4, 16-21.

42. Vinayaka, K.S.; Swarnalatha, S.P.; Preethi, H.R.; Surabhi, K.S.; Prashith Kekuda, T.R.; Sudharshan, S.J. Studies on in vitro antioxidant, antibacterial and insecticidal activity of methanolic extract of Abrus pulchellus Wall (Fabaceae). Afr. J. Basic Appl. Sci. 2009, 1, 110-116.

43. Mattila, P.; Vaananen, P.S.; Kongo, K.; Aro, H.; Jalava, T. Basic composition and amino acid contents of mushrooms cultivated in Finland. J. Agric. Food Chem. 2002, 50, 6419-6422. 
44. Barros, L.; Ferreira, M.J.; Queiro's, B.; Ferreira, I.C.F.R.; Baptista, P. Total phenols, ascorbic acid, b-carotene and lycopene in Portuguese wild edible mushrooms and their antioxidant activities. Food Chem. 2007, 103, 413-419.

45. Mau, J.L.; Lin, H.C.; Chen, C.C. Antioxidant properties of several medicinal mushrooms. J. Agric. Food Chem. 2002, 50, 6072-6077.

46. Wu, X.J.; Hansen, C. Antioxidant capacity, phenolic content, and polysaccharide content of Lentinus edodes grown in whey permeate-based submerged culture. J. Food Sci. 2010, 73, 1-8.

47. Lee, T.T.; Huang, C.C.; Shieh, X.H.; Chen, C.L.; Chen, L.J.; Yu, B.I. Flavonoid, phenol and polysaccharide contents of Echinacea purpurea L. and its immunostimulant capacity in vitro. Int. J. Environ. Sci. Dev. 2010, 1, 5-9.

48. Kontush, A.; Finckh, B.; Karten, B.; Kohlschütter, A.; Beisiegel, U. Antioxidant and prooxidant activity of alpha-tocopherol in human plasma and low density lipoprotein. J. Lipid Res. 1996, 37, 1436-1448.

49. Builders, P.F.; Ezeobi, C.R.; Tarfa, F.D.; Builders, M.I. Assessment of the intrinsic and stability properties of the freeze-dried and formulated extract of Hibiscus sabdariffa Linn. (Malvaceae). Afr. J. Pharm. Pharmacol. 2010, 4, 304-313.

50. Knežević, S.V.; Blažeković, B.; Štefan, M.B.; Alegro, A.; Köszegi, T.; Petrik, J. Antioxidant activities and polyphenolic contents of three selected Micromeria species from Croatia. Molecules 2011, 16, 1454-1470.

51. Barros, L.; Baptista, P.; Estevinho, L.M.; Ferreira, I.C.F.R. Effect of fruiting body maturity stage on chemical composition and antimicrobial activity of Lactarius sp. mushrooms. J. Agric. Food Chem. 2007, 55, 8766-8771.

52. Barros, L.; Calhelha, R.C.; Vaz, J.A.; Ferreira, I.C.F.R.; Baptista, P.; Estevinho, L.M. Antimicrobial activity and bioactive compounds of Portuguese wild edible mushrooms methanolic extracts. Eur. Food Res.Technol. 2007, 225, 151-156.

53. Altaf, S.A.; Umar, D.M.; Muhammad, M.S. Production of xylanase enzyme by Pleurotus eryngii and Flamulina velutipes grown on different carbon sources under submerged fermentation. World Appl. Sci. J. 2010, 8, 47-49.

54. Naraian R.; Naveen K.A.; Garg S.K. Improved submerged fermentation of corn cob with mechanically broken oil seed cakes and decolorisation of textile dyes by enzyme extract of Pleurotus florida PF05. Res. Environ. Life Sci. 2009, 2, 83-90.

55. Oboh, I.E.; Akerele, J.O; Obasuyi, O. Antimicrobial activity of the ethanol extract of the aerial parts of Sida acuta burm.f. (malvaceae). Trop. J. Pharm. Res. 2007, 6, 809-813.

56. Sreenivasan, S.; Darah, I.; Mohd, M.J.N.K. Free radical scavenging activity and total phenolic compounds of Gracilaria changii. Int. J. Eng. Sci. 2007, 1, 115-117.

57. Sheng, Z.W.; Ma, W.H.; Gao, J.H.; Bi, Y.; Zhang, W.M.; Dou, H.T.; Jin, Z.Q. Antioxidant properties of banana flower of two cultivars in China using 2,2-diphenyl-1-picrylhydrazyl (DPPH,) reducing power, 2,2'-azinobis-(3-ethylbenzthiazoline-6-sulphonate (ABTS) and inhibition of lipid peroxidation Assays. Afr. J. Biotechnol. 2011, 10, 4470-4477.

58. Sokmen, A.; Gulluce, M.; Akpulat, H.A.; Daferera, D.; Tepe, B.; Polissiou, M.; Sokmen, M.; Sahin, F. The in vitro antimicrobial and antioxidant activities of the essential oils and methanol extracts of endemic Thymus spathulifolius. Food Control 2007, 15, 627-634. 
59. Zengin, G.; Aktumsek, A.; Guler, G.O.; Cakmak, Y.S.; Yildiztugay, E. Antioxidant properties of methanolic extract and fatty acid composition of Centaurea urvillei DC. subsp. hayekiana Wagenitz. Rec. Nat. Prod. 2011, 5, 123-132.

60. Gulcin, I.; Buyukokuroglu, M.E.; Oktay, M.; Kufrevioglu, O.I. Antioxidant and analgesic activities of turpentine of Pinus nigra Arn. subsp. pallsiana (Lamb.) Holmboe. J. Ethnopharmacol. 2003, 86, 51-58.

61. Chou, H.J.; Kuo, J.T.; Lin, E.S. Comparative antioxidant properties of water extracts from different parts of Beefsteak plant (Perilla frutescens). J. Food Drug Anal. 2009, 17, 489-496.

62. Lin, E.S.; Li, C.C. Evaluation of superoxide radical scavenging capacity and reducing power of areca flower extracts. J. Med. Plants Res. 2010, 4, 975-981.

63. Varshneya, C.; Varshneya, C.; Kant, V.; Mehta, M. Total phenolic contents and free radical scavenging activities of different extracts of seabuckthorn (Hippophae rhamnoides) pomace without seeds. Int. J. Food Sci. Nutr. 2012, 63, 153-159.

64. Hazra, B.; Biswas, S.; Mandal, N. Antioxidant and free radical scavenging activity of Spondias pinnata. BMC Complement. Altern. Med. 2008, 8, 63.

65. Sunil, K.; Dinesh, K.M.; Kamal, S.; Nidhan, S.; Bhoodev, V. Antioxidant and free radical scavenging potential of Citrullus colocynthis (L.) Schrad. methanolic fruit extract. Acta Pharm. 2008, 58, 215-220.

66. Anesini, C.; Ferraro, G.E.; Filip, R. Total polyphenol content and antioxidant capacity of commercially available tea (Camellia sinensis) in Argentina. J. Agric. Food Chem. 2008, 56, 9225-9229.

67. Kim, I.S.; Yang, M.R.; Lee, O.H.; Kang, S.N. Antioxidant activities of hot water extracts from various spices. Int. J. Mol. Sci. 2011, 12, 4120-4131.

68. Meda, A.; Lamien, C.E.; Romito, M.; Millogo, J.; Nacoulma, O.G. Determination of the total phenolic, flavonoid and proline contents in Burkina Fasan honey, as well as their radical scavenging activity. Food Chem. 2005, 91, 571-577.

69. Kivcak, B.; Akay, S. Quantitative determination of a-tocopherol in Pistacia lentiscus, Pistacia lentiscus var. chia, and Pistacia terebinthus by TLC-densitometry and colorimetry. Fitoterapia 2005, 76, 62-66.

Sample Availability: Not available.

(C) 2012 by the authors; licensee MDPI, Basel, Switzerland. This article is an open access article distributed under the terms and conditions of the Creative Commons Attribution license (http://creativecommons.org/licenses/by/3.0/). 